\title{
Interparticle Spacing Control in the Superlattices of Carboxylic Acid-Capped Gold Nanoparticles by Hydrogen-Bonding Mediation
}

Hiroshi Yao, ${ }^{*}$ Hiroyuki Kojima, Seiichi Sato, and Keisaku Kimura

Graduate School of Material Science, University of Hyogo, 3-2-1 Koto, Kamigori-cho, Ako-gun, Hyogo

678-1297, Japan

\section{Transmission Electron Microscope (TEM) Image}

TEM image of a 2D superlattice of gold nanoparticles in thæyA-SL sample. The observation area is different from that shown in Figure 3a. This image represents the variation in the size of the nanoparticles in the superlattice. The mean core diameter of nanoparticles at the left side in the image $(4-4.5 \mathrm{~nm})$ is apparently larger than that at the right side (3-3.5 $\mathrm{nm})$.

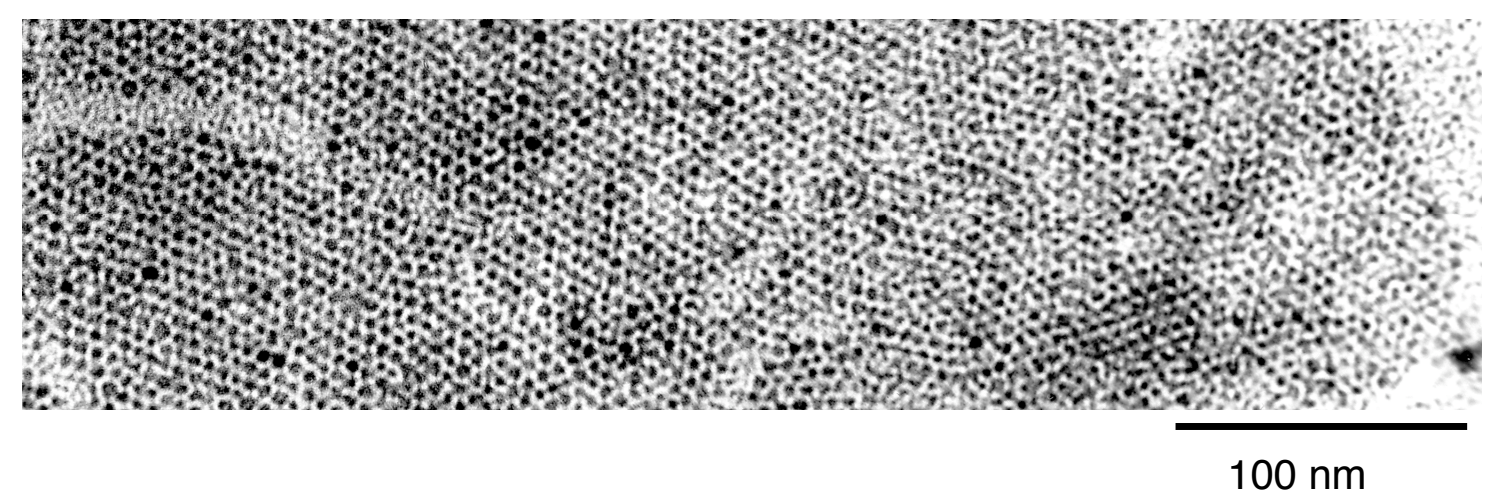

Figure S1. TEM image of a 2D superlattice of nanoparticles (PyA-SL film). The observation area is different from that shown in Figure $3 \mathrm{a}$. 